\title{
Superior Water Maze Performance and Increase in Fear-Related Behavior in the Endothelial Nitric Oxide Synthase-Deficient Mouse Together with Monoamine Changes in Cerebellum and Ventral Striatum
}

\author{
Christian Frisch, ${ }^{1}$ Ekrem Dere, ${ }^{1}$ Maria Angelica De Souza Silva, ${ }^{1}$ Axel Gödecke, ${ }^{2}$ Jürgen Schrader, ${ }^{2}$ and \\ Joseph P. Huston ${ }^{1}$
}

1/nstitute of Physiological Psychology and 2Institute of Physiology, Center for Biological and Medical Research, University of Düsseldorf, D-40225 Düsseldorf, Germany

\begin{abstract}
Nitric oxide (NO) has been implicated in the control of emotion, learning, and memory. We have examined endothelial NO synthase-deficient mice (eNOS-/-) in terms of habituation to an open field, elevated plus-maze behavior, Morris water maze performance, and changes in cerebral monoamines. In the open field, eNOS - / - animals were less active than wild-type controls but showed unimpaired habituation. In the plus-maze, an anxiogenic effect was observed. Proceeding from previous findings of deficits in hippocampal and neocortical long-term potentiation (LTP) in our eNOS-/- mice, we investigated whether these animals also express deficits in learning tasks that have been linked to hippocampal function and LTP. Unexpectedly, eNOS gene disruption led to accelerated place learning in the water maze. Furthermore, during long-term retention and reversal learning, eNOS - / - mice showed improved performance. In a cued version of the water maze task, eNOS -/- and control mice
\end{abstract}

Nitric oxide (NO) is involved in neurosynaptic transmission (Manzoni et al., 1992; Montague et al., 1994) and plays an important role in the control of cerebral blood flow (Faraci and Brian, 1994). The brain distribution of NO synthases (NOS) has been investigated in some detail (Kidd et al., 1995; Hara et al., 1996). NOS-like activity was found in regions involved in activity, anxiety, and memory, such as the hippocampus and the amygdala (Dinerman et al., 1994; Dun et al., 1994). Pharmacological inhibition of NO synthesis reduced spontaneous and stimulant-induced activity (Ohno and Watanabe, 1995; Sandi et al., 1995), had anxiogenic effects (Lino de Oliveira et al., 1997), and diminished the effects of anxiolytic agents (Quock and Nguyen, 1992; Caton et al., 1994). The role of $\mathrm{NO}$ in learning and memory is unclear. NOS inhibition was reported to slow down (Chapman et al., 1992), as well as to accelerate (Du and Harvey, 1996), classical conditioning. Application of an NO donator improved or impaired avoidance learning, depending on dose (Huang and Lee, 1995). In the water maze, systemic NO inhibition had deleterious effects in some studies (Chapman et al., 1992; Estall et al., 1993; Yamada et al., 1995), whereas other work indicates no effects of systemic or subtotal hippocampal inhibition of NO synthesis (Bannerman et al., 1994; Blokland et al., 1999).

Genetic inactivation of endothelial but not of neuronal NOS (nNOS) led to a strong reduction in NMDA-induced GABA re-

Received Feb. 22, 2000; revised May 31, 2000; accepted June 13, 2000.

This work was supported by the Center for Biological and Medical Research, University of Düsseldorf, Germany.

Correspondence should be addressed to Joseph P. Huston, Institute of Physiological Psychology, University of Düsseldorf, Universitätstraße 1, 40225 Düsseldorf, Germany. E-mail: huston@uni-duesseldorf.de.

Copyright (C) 2000 Society for Neuroscience $0270-6474 / 00 / 206694-07 \$ 15.00 / 0$ did not differ, implying that the superior performance of eNOS-/- animals on the former tasks cannot be attributed solely to differences in sensorimotor capacities. The neurochemical evaluation of the eNOS $-/-$ mice revealed increases in the concentrations of the serotonin metabolite 5-HIAA in the cerebellum, together with an accelerated serotonin turnover in the frontal cortex. Furthermore, eNOS-/- mice had a higher dopamine turnover in the ventral striatum. These findings are discussed in terms of possible concomitant effects on physiological parameters, such as a decreased reactivity of GABAergic neurotransmission or changes in vascular functions, and effects on behavioral processes related to reinforcement, learning, and emotion.

Key words: endothelial nitric oxide synthase; mouse mutants; genetic inactivation; anxiety; activity; learning; memory; serotonin; dopamine; cerebellum; ventral striatum lease in several brain regions, including the hippocampus, whereas NMDA-induced glutamate release was reduced only by the inactivation of the neuronal isoform (Kano et al., 1998). Endothelial NOS (eNOS) and nNOS show distinct expression patterns in the hippocampus (Son et al., 1996) in which eNOS- but not nNOSderived NO is involved in long-term potentiation (LTP) (O`Dell et al., 1994). Considering these differential neurophysiological roles of the NOS isoforms, their behavioral functions might also differ. For example, aggressive behavior was diminished in eNOS-deficient (eNOS $-/-$ ) mice, whereas it was increased in nNOS-deficient animals (Demas et al., 1999). In the present study, to gauge whether the effects of inactivation of eNOS on emotional and motor behavior are comparable with those found after pharmacological inhibition of NO, we examined eNOS $-/-$ mice behavior in the elevated plus-maze and during repeated exposure to an open field. In the water maze, we subjected the animals to a sequence of tasks that gauge different aspects of learning, such as spatial orientation, long-term retention, reversal learning, and stimulus-oriented learning. Given the LTP deficits reported for our eNOS $-/-$ mice (Wilson et al., 1997; Haul et al., 1999) and given that the traditional view of a positive functional relationship between LTP and learning capacity holds true, we expected to find impaired water maze performance in eNOS $-1-$ mice.

Monoaminergic neurons are crucially involved in the control of behavioral processes related to exploration, anxiety, learning, and memory (Barnes and Sharp, 1999; Rolls, 2000). Pharmacological manipulation of the NO system influences cerebral monoaminergic activity (Montague et al., 1994; Yamada et al., 1995). Therefore, we expected to find changes in the concentrations of noradrenaline, dopamine (DA), serotonin (5-HT), and their metabolites in the eNOS-deficient brain. 


\section{MATERIALS AND METHODS}

\section{Animals}

eNOS $-/-$ mice ( 3 months of age) were backcrossed for seven generations into the $\mathrm{C} 57 \mathrm{BL} / 6$ genetic background. Age-matched eNOS $+/+$ mice aswell as C57BL/6 mice served as controls. Eight eNOS $-/-$ mice and six eNOS $+/+$ mice [wild type (WT)] were tested in the elevated plus-maze, followed by the open field. In the water maze, new batches of 24 eNOS - / mice, 8 eNOS $+/+$ mice, and 16 C57BL $/ 6$ mice were tested. Behavioral measures for the latter two groups did not differ, so that they were combined (WT). Generation and functional characterization of eNOS $-/-$ mice have been described previously (Gödecke et al., 1998). The animals were maintained on a normal $12 \mathrm{hr}$ light/dark cycle and were tested during the light phase. They were housed singly in standard Makrolon cages with sawdust bedding for 2 weeks before the beginning of the experiments and had access to food (10H10; Nohrlin, Bad Salzuflen, Germany) and tap water ad libitum.

\section{Elevated plus-maze}

The plus-maze consisted of two open arms $(38.5 \times 5 \mathrm{~cm})$ and two walled arms $(38.5 \times 5 \times 15 \mathrm{~cm})$ with an open roof, arranged around the central platform $(5 \times 5 \mathrm{~cm})$ so that the two arms of each type were opposite to each other. The maze was elevated to a height of $40 \mathrm{~cm}$. A video camera, a loudspeaker providing masking noise, and a $25 \mathrm{~W}$ red light bulb placed 250 $\mathrm{cm}$ above the maze (illumination density at the center of the maze, 0.3 lux) were positioned above its center. The digitized image of the path taken by each animal was stored and analyzed post hoc with a semi-automated analysis system (Ethovision, Noldus, The Netherlands). After each trial, the apparatus was swept out with water containing $0.1 \%$ acetic acid. The rats were placed on the central platform of the maze facing one of the walled arms and were observed for $5 \mathrm{~min}$, during which the number of entries into and time spent in the open and enclosed arms were measured. Furthermore, the frequency of line crossings on the walled arms was determined by dividing both arms by two virtual lines into three sections of equal size, counting the total number of crossings of these lines, and dividing the score by the time spent on the walled arms. On the elevated plus-maze, rodents display a variety of fear-related behaviors in addition to the avoidance of the open arms (Cruz et al., 1994). To examine the "anxiolytic profile" exhibited by the eNOS-deficient mice, scanning (protruding the head over the edge of an open arm and fanning with the vibrissae in any direction), risk assessment (protruding from an enclosed arm with the forepaws and head only), and end activity (number and amount of time spent at the end of an open arm) were determined.

\section{Open field}

The open-field apparatus was a rectangular chamber $(60 \times 60 \times 40 \mathrm{~cm})$ made of gray polyvinyl chloride. The video taping, illumination, and masking noise arrangement was the same as for the plus maze experiment. The behavioral parameters registered during $5 \mathrm{~min}$ sessions were as follows: (1) rearing, the number of times an animal was standing on its hind legs with forelegs in the air or against the wall; (2) locomotion, the distance in centimeters an animal moved; and (3) corner time, the time spent in the four corner squares $(10 \times 10 \mathrm{~cm})$. The animals were reexposed to the open field 24 and $96 \mathrm{hr}$ after the initial trial.

\section{Water maze}

Apparatus. The water maze used consisted of a black circular tank, $110 \mathrm{~cm}$ in diameter and with a wall $40 \mathrm{~cm}$ in height. It was filled to a depth of $25 \mathrm{~cm}$ with water $\left(19-20^{\circ} \mathrm{C}\right)$ made opaque-white by the addition of milk powder. The escape platform, made of white Plexiglas, had a diameter of $10 \mathrm{~cm}$ and was height-adjustable. Swimming paths were stored and analyzed post hoc with the system used for the elevated plus-maze and open-field sessions. The room was illuminated by ceiling lamps. A number of obvious distal cues available during water maze testing included doors, racks, and ceiling texture.

Habituation (day1). During a habituation trial with no platform placed in the pool, the animals had to swim for $60 \mathrm{sec}$.

Acquisition (days 2-10). Beginning on the following day, the mice were tested in the place version of the task for $8 \mathrm{~d}$ ("place learning," days 2-9). For each animal, the platform was submerged $0.5 \mathrm{~cm}$ beneath the water surface in one of the four quadrants of the maze, where it remained during all place learning trials. Mice were placed into the maze from four equally spaced points along the perimeter of the pool. The sequence of entry points was chosen randomly. On every day, each mouse received four trials (cutoff, $60 \mathrm{sec}$ ). After reaching the platform, the animals were allowed to stay on it for $30 \mathrm{sec}$. If an animal failed to escape within $60 \mathrm{sec}$, it was placed onto the platform for $30 \mathrm{sec}$. During the $60 \mathrm{sec}$ intertrial interval, the animals were placed into a resting cage beside the pool. On a subsequent trial in which the platform was removed from the pool ("spatial probe" or extinction trial, day 10), the mice had to swim again for $60 \mathrm{sec}$ with no opportunity to escape.

Long-term retention (days 15-16). Five days after the extinction trial, the animals were tested over $2 \mathrm{~d}$ for long-term retention of the platform position with the platform placed at its initial position during place learning. Retention testing was performed for $2 \mathrm{~d}$ using the same daily procedure as during place learning.

\section{A. Time in Compartments}

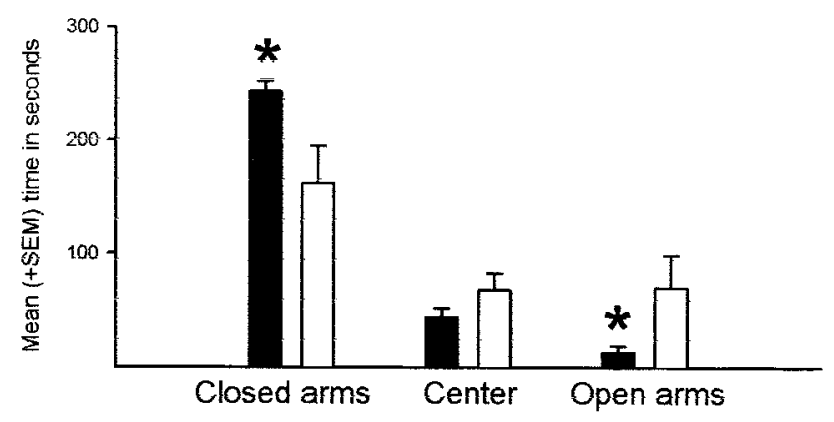

B. Activity

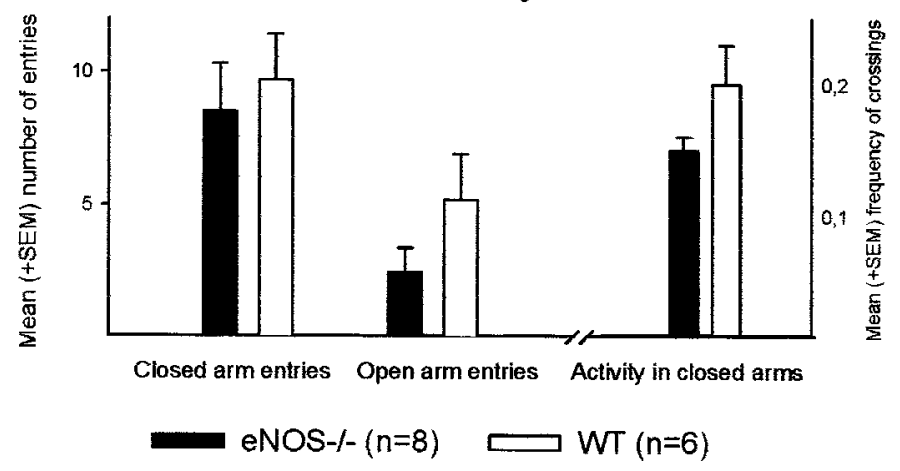

Figure 1. Elevated plus-maze. Effects of the eNOS gene inactivation on behavior in the elevated plus-maze. $A$, Mean + SEM time in seconds spent in the closed arms, center, and on the open arms. $B$, Mean + SEM number of entries into the enclosed (left) and open arms (center), and mean + SEM number of crossings in the closed arms (right) of the plus-maze. ${ }^{*} p<0.05$ ( $t$ test) versus WT controls.

Reversal (days 17-20). Beginning on the day after retention testing, animals were trained to find the platform at a new position in the quadrant opposite to its original location. This reversal training was performed for $4 \mathrm{~d}$ with the same procedure as described for original place learning and long-term retention testing.

Cued version (days 21-22). One day after retention testing, the platform, made visible by a cylinder (diameter of $2 \mathrm{~cm}$, height of $10 \mathrm{~cm}$ ) with black and white stripes, was placed at a position not used during the former sessions. Testing was conducted for another $2 \mathrm{~d}$ with the same procedure.

\section{Neurochemical analysis}

After the end of behavioral testing in the water maze, seven eNOS $-/-$ and seven WT mice representative of their populations underwent postmortem neurochemical analysis. These animals were decapitated, their brains were quickly removed, and the medial frontal cortex, ventral striatum, neostriatum, hippocampus, and the cerebellum were dissected out bilaterally on ice. These tissue samples were analyzed for 5-HT, 5-hydroxyindole acetic acid (5-HIAA), noradrenaline, dopamine, dihydrophenylacetic acid (DOPAC), and homovanillic acid (HVA) levels using HPLC with electrochemical detection (for technical details, see De Souza Silva et al., 1997).

\section{Statistical analysis}

For the analysis of behavioral and neurochemical data, $t$ tests (two-tailed) for dependent and independent measures were used. Furthermore, water maze data were analyzed with ANOVA procedures (blocks of four daily trials for repeated measures).

\section{RESULTS}

\section{Elevated plus-maze}

Compared with WT mice $(n=6)$, the eNOS $-/-$ animals $(n=8)$ spent less time on the open arms ( $t$ test, $p=0.044)$ (Fig. 1$)$ and more time on the closed arms $(p=0.016)$. The number of entries into the arms and the frequency of line-crossings were unchanged (entries into arms, $p=0.290$; line crossings, $p=0.104$ ). Frequency but not time spent in end activity was lower in eNOS $-/-$ animals 
Table 1. Frequency and duration of scanning, risk assessment, and end activity during the $5 \mathrm{~min}$ test period in the elevated plus-maze for eNOS-/- and WT mice

\begin{tabular}{|c|c|c|c|c|}
\hline & & Scanning & Risk assessment & End activity \\
\hline & & Mean \pm SEM & Mean \pm SEM & Mean + SEM \\
\hline WT & $\mathrm{F}$ & $14.00 \pm 5.74$ & $7.17 \pm 2.01$ & $4.00 \pm 1.57$ \\
\hline & $\mathrm{T}$ & $19.10 \pm 8.47$ & $25.52 \pm 9.26$ & $30.26 \pm 13.85$ \\
\hline $\mathrm{KO}$ & $\mathrm{F}$ & $2.50 \pm 0.91^{*}$ & $11.63 \pm 1.29$ & $0.63 \pm 0.42 *$ \\
\hline & $\mathrm{T}$ & $2.27 \pm 1.17^{*}$ & $44.02 \pm 6.95$ & $5.03 \pm 3.78$ \\
\hline
\end{tabular}

F, Frequency; T, time (in seconds). ${ }^{*} p<0.05$ ( $t$ test) versus WT controls.

[frequency $(\mathrm{F}), p=0.035$; time $(\mathrm{T}), p=0.067$ ] (Table 1). Furthermore, in eNOS $-/-$ mice, frequency and duration of scanning were reduced $(\mathrm{F}, p=0.046 ; \mathrm{T}, p=0.041)$, whereas frequency of risk assessment showed a tendency to increase $(p=0.074)$. Duration of risk assessment was unchanged $(p=0.128)$. This behavioral profile indicates an anxiogenic effect of the eNOS gene disruption.

\section{Open field}

ENOS $-/-$ mice $(n=8)$ showed less activity in the open field than wild-type controls $(n=6)$. During the first and third open-field sessions, the total distance moved by the eNOS $-/-$ animals was less (first session, $p=0.040$; third session, $p=0.042$ ) (Fig. $2 A$ ), whereas no difference was detected during the second session $(p=$ 0.328). Furthermore, eNOS $-/-$ mice showed less rearing during the third trial (first session, $p=0.123$; second session, $p=0.141$; third session, $p=0.013$ ) (Fig. $2 B$ ). Furthermore, the eNOS $-/-$ mice spent more time in the corners of the apparatus during the first session $(p=0.034)$ (Fig. $2 C)$. This pattern of results indicates an increase in anxiety-related avoidance of open space and thereby confirms the results of the plus-maze experiment.

Both groups showed signs of habituation to the new environment, as indicated by a tendency for a decrease in the total distance moved by eNOS $-/-$ mice $(p=0.055)$, a decrease shown by the control mice $(p=0.014)$, and a reduction in rearing in both groups (eNOS mice, $p=0.042$; control mice, $p=0.020$ ) from the first to the second session. The time spent in the corners increased from the first to the second session (eNOS $-/-$ mice, $p=0.035$; control mice, $p=0.030)$. Control but not knock-out (KO) animals showed an increase in the distance moved from the second to the third session (control mice, $p=0.004$; eNOS $-/-$ mice, $p=0.207$ ), possibly indicative of superior habituation learning by the eNOS $-1-$ mice.

\section{Water maze}

\section{Habituation (day 1)}

During the habituation trial, no impairments in swimming capability were observed in eNOS-deficient animals $(n=24)$ compared with wild-type controls $(n=24)$. In fact, the eNOS $-/-$ mice swam a longer distance than wild-type animals ( $t$ test, $p=0.029)$. Platform quadrant time and time spent in the other quadrants was comparable within and between groups ( $p$ values $>0.1)$.

\section{Acquisition (place learning, days 2-9)}

During initial place learning, the mice learned to find the hidden platform, as indicated by an effect of time on search latencies (ANOVA, trial blocks, $F_{(7,322)}=67.4, p<0.001$ ) (Fig. $3 A$ ). eNOS-deficient mice found the platform faster than control animals, as indicated by an effect of genotype on search time $\left(F_{(1,46)}=\right.$ $5.35, p=0.025)$. There was no interaction between genotype and training $(p>0.1)$. Furthermore, there was no effect of genotype on the distance moved until the platform was reached or on swimming speed (distance moved, $F_{(1,46)}=0.70, p=0.408$; Fig. $3 B$ ) (swimming speed, $F_{(1,46)}=0.72, p=0.196$; Fig. $\left.3 C\right)$.

\section{Extinction trial (day 10)}

Both groups showed a clear preference for the former platform quadrant (ANOVA, eNOS $-/-$ mice, $F_{(3,69)}=28.2, p<0.001$;
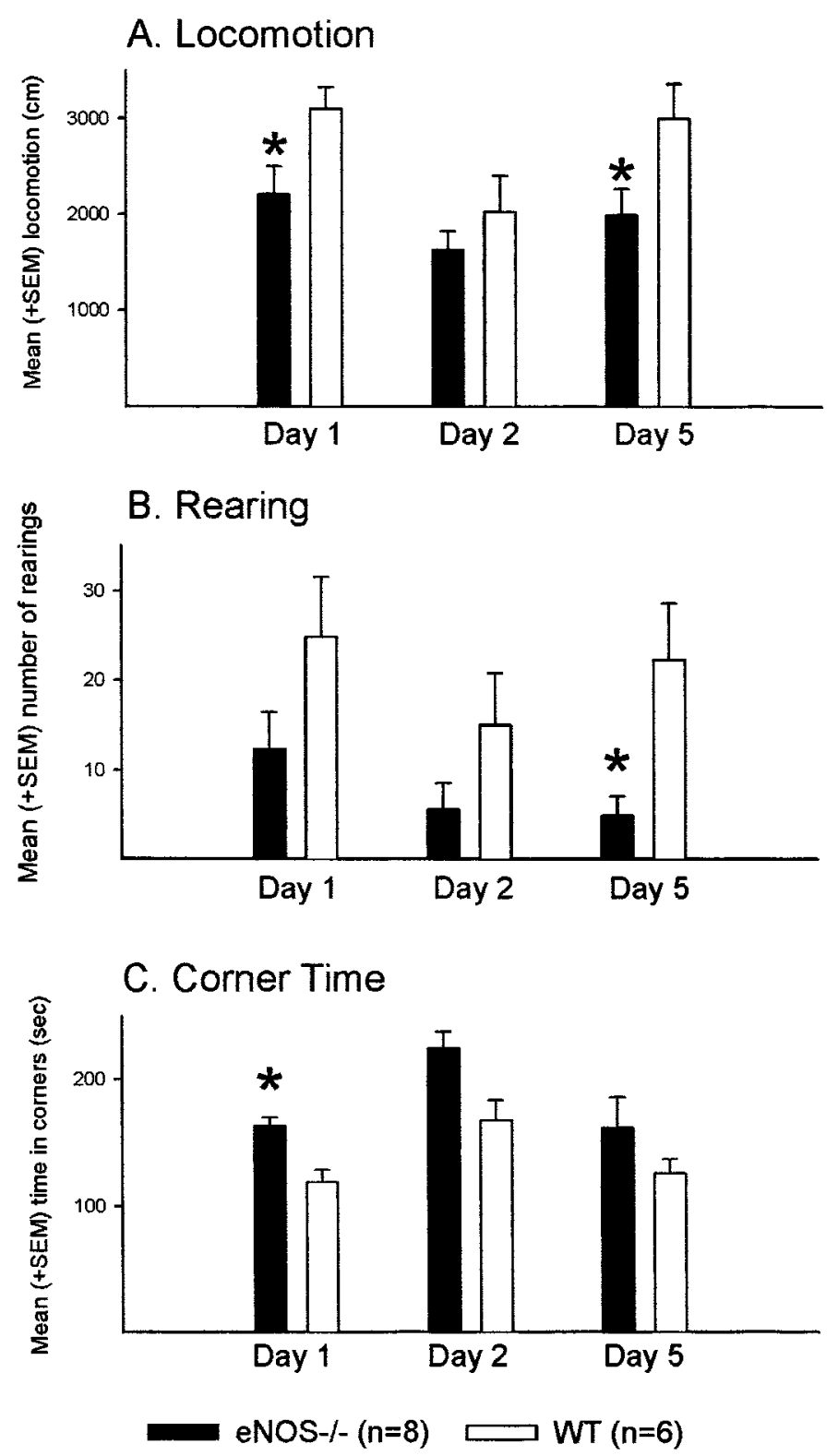

Figure 2. Open field. Effects of the eNOS gene disruption on behavior in the open field during three 5 min exposures on indicated days. $A$, Mean + SEM locomotion in centimeters. $B$, Mean + SEM number of rearings. $C$, Mean + SEM time spent in the four corners of the apparatus. ${ }^{*} p<0.05$ $(t$ test) versus WT controls during the respective day of testing.

$\left.\mathrm{WT}, F_{(3,69)}=28.7, p<0.001\right)($ Fig. $4 A)$. There were no significant between-group differences in time spent in the platform quadrant ( $t$ test, $p=0.811$ ) but a tendency for an increase in the number of platform position crossings by the eNOS $-/-$ mice $(p=0.070)$ (Fig. $4 B$ ). Furthermore, mean swimming speed during the $60 \mathrm{sec}$ testing session was comparable between groups $(p=0.291)$ (Fig. 4C).

\section{Long-term retention (days 15-16)}

Five days after the extinction trial, the animals were tested again with the platform submerged at its original location. During the course of retention learning, eNOS mice found the platform sooner than wild-type controls (ANOVA, $F_{(1,46)}=12.1, p=0.001$ ) (Fig. $3 A$ ). Again, there was an effect of training on latencies (trial blocks, $\left.F_{(1,46)}=6.70, p=0.013\right)$ but no interaction between genotype and training $(p>0.1)$. Here, in contrast to the initial learning during acquisition, an effect of genotype on the distance moved and on swim speed was observed. eNOS-deficient mice swum a shorter 


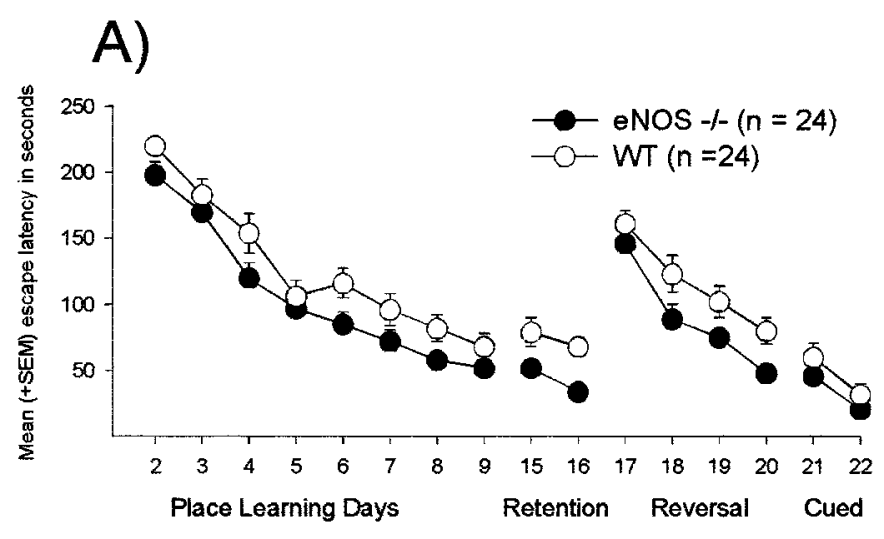

B)
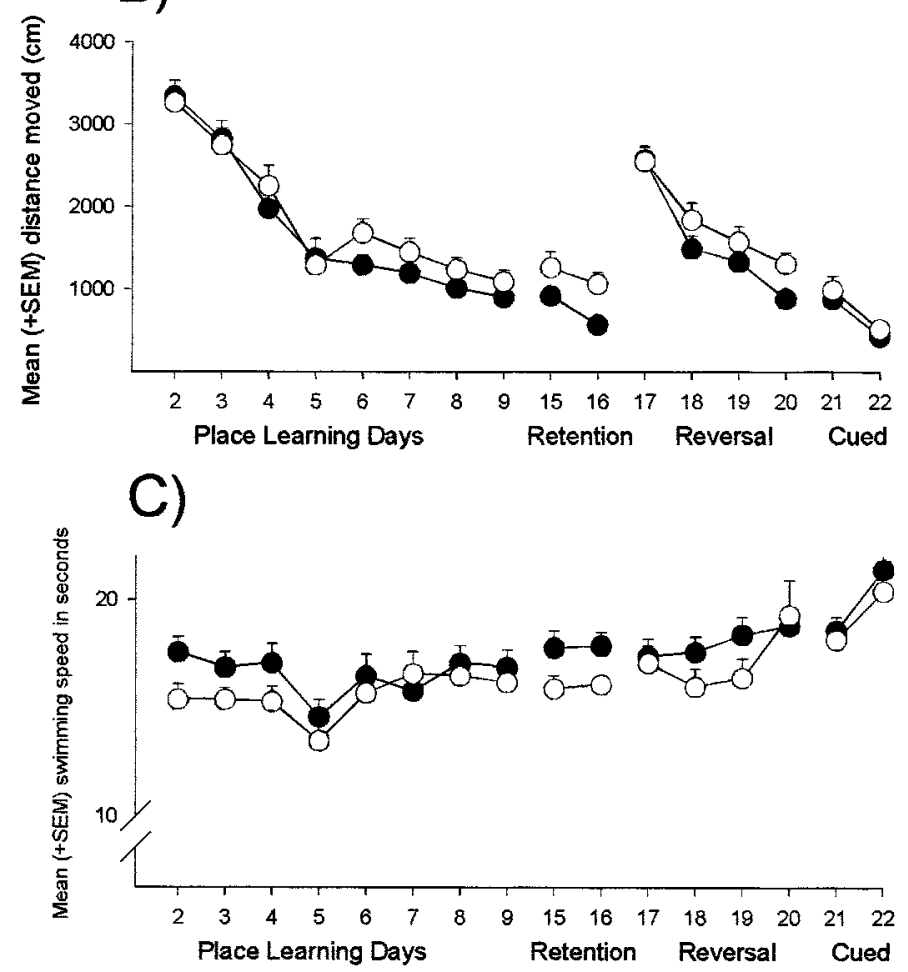

Figure 3. Water maze. Effects of the eNOS gene disruption on acquisition, retention, reversal, and cued learning performance in the water maze. $A$, Mean + SEM escape latencies. $B$, Mean + SEM path length. $C$, Mean + SEM swimming speed.

distance until the platform was reached with a higher velocity (distance moved, $F_{(1,46)}=7.29, p=0.010$; Fig. $3 B$ ) (swimming speed, $F_{(1,46)}=5.13, p=0.028$; Fig. $3 C$ ). Furthermore, single trial analysis revealed that eNOS $-/-$ mice showed shorter escape latencies than controls during the first retention trial on day 15 and during the first three trials on day 16 ( $t$ test, trial 1 , day $15, p=$ 0.023 ; trial 1 , day $16, p=0.001$; trial 2 , day $16, p=0.015$; trial 3 , day $16, p=0.041$ ) (Fig. 5).

\section{Reversal (days 17-20)}

During the course of reversal training, the eNOS $-/-$ mice again showed superior performance (ANOVA, $F_{(1,46)}=5.98, p=0.018$ ) (Fig. $3 A)$. Escape latencies decreased over time $\left(F_{(3,138)}=44.6, p<\right.$ $0.001)$. Again, no interaction between genotype and training was observed $(p>0.1)$. No effect of genotype on the distance moved or on swimming speed was observed (distance moved, $F_{(1,46)}=$ 2.47, $p=0.123$; Fig. $3 B$ ) (swimming speed, $F_{(1,46)}=0.64, p=0.429$; Fig. $3 C$ ). Single-trial analysis revealed that, during the second trial on day 19 and the third trial on day 20 , eNOS $-/-$ mice reached the

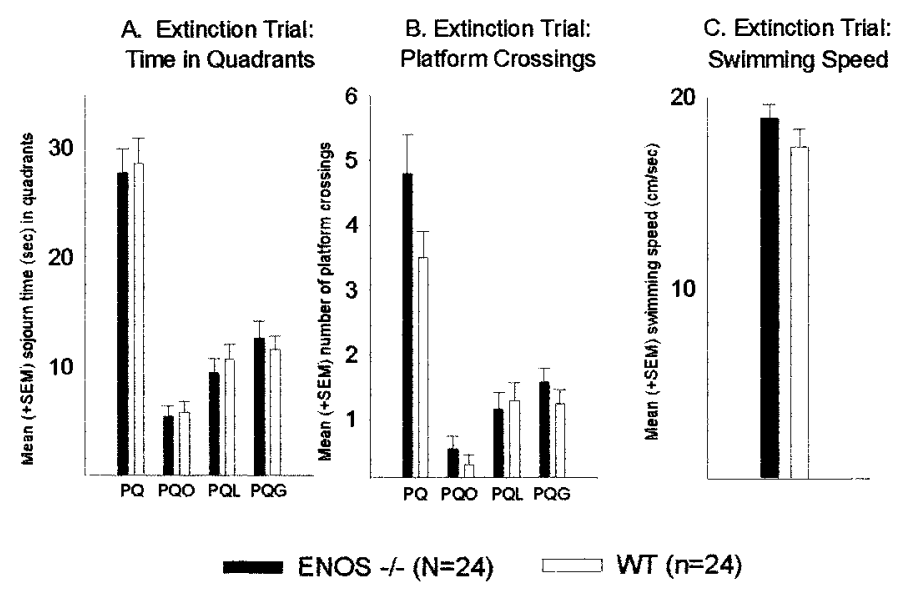

Figure 4. Water maze: extinction trial. Effects of the eNOS gene inactivation on extinction behavior in the water maze. $A$, Mean + SEM sojourn time (in seconds). $B$, Mean + SEM number of platform position crossings. $C$, Mean + SEM swimming speed (in centimeters per second) during extinction trial in the four quadrants of the maze. $P Q$, Platform quadrant; $P Q O$, quadrant opposite to $\mathrm{PQ} ; P Q L$, quadrant left from $\mathrm{PQ} ; P Q R$, quadrant right from $\mathrm{PQ}$.

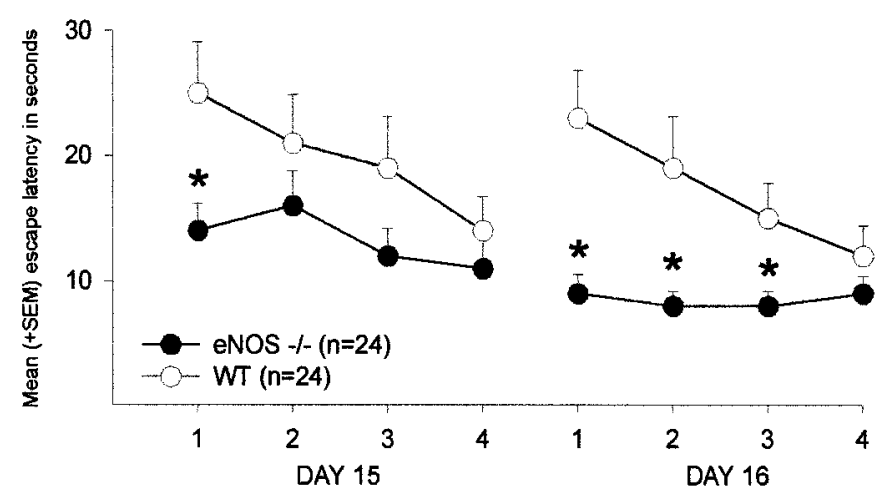

Figure 5. Water maze: retention. Effects of the eNOS gene inactivation on single-trial retention performance in the water maze. Depicted are the mean + SEM escape latencies (in seconds) during every retention trial on days 15 and $16 .{ }^{*} p<0.05$ ( $t$ test) versus WT controls.

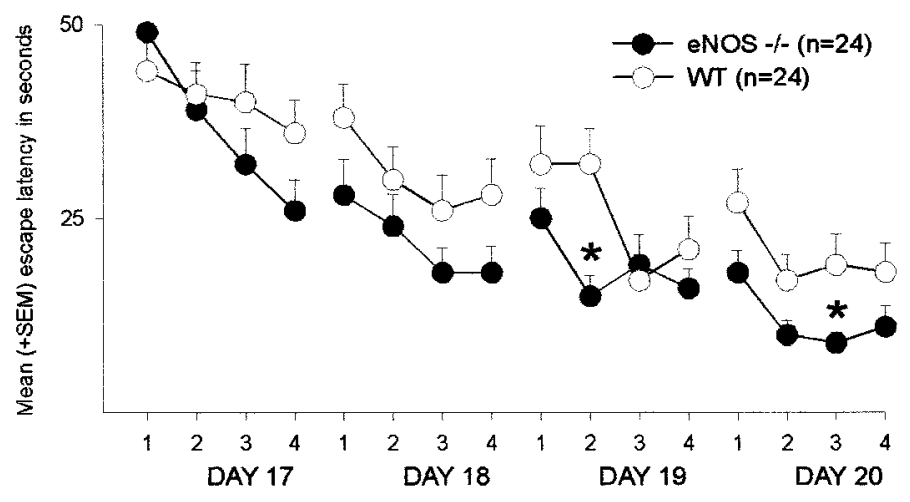

Figure 6. Water maze: reversal. Effects of the eNOS gene disruption on single-trial performance during reversal learning in the water maze. Depicted are mean + SEM escape latencies (in seconds) during every reversal trial on days $17-20$. * $p<0.05$ ( $t$ test) versus WT controls.

platform faster $(t$ test, tria1 2, day 19, $p=0.001$; trial 3, day 20, $p=$ 0.019) (Fig. 6).

Cued version (days 21-22)

During testing with the visible platform, between-group differences in escape latency were not observed (ANOVA, $F_{(1,46)}=1.88, p=$ 0.177 ) (Fig. $3 A$ ). Performance improved during the course of cued 


\begin{tabular}{|c|c|c|c|c|c|c|c|c|c|}
\hline & NA & DA & HVA & DOPAC & $5-\mathrm{HT}$ & 5-HIAA & HVA/DA & DOPAC/DA & 5-HIAA/5-HT \\
\hline \multicolumn{10}{|l|}{ Cerebellum } \\
\hline WT & $54.86 \pm 2.68$ & $3.18 \pm 0.21$ & $1.18 \pm 0.12$ & n.d. & $13.22 \pm 1.18$ & $7.11 \pm 0.60$ & $0.62 \pm 0.13$ & & $0.64 \pm 0.04$ \\
\hline eNOS-l- & $59.40 \pm 6.10$ & $2.49 \pm 0.33^{+}$ & $1.36 \pm 0.07$ & n.d. & $14.21 \pm 1.02$ & $8.92 \pm 0.32 *$ & $0.38 \pm 0.06^{+}$ & & $0.56 \pm 0.06$ \\
\hline \multicolumn{10}{|l|}{ Frontal cortex } \\
\hline WT & $69.86 \pm 6.65$ & $10.84 \pm 0.43$ & $7.54 \pm 1.46$ & n.d. & $40.81 \pm 3.44$ & $9.09 \pm 1.51$ & $0.71 \pm 0.04$ & & $0.22 \pm 0.03$ \\
\hline eNOS-/- & $80.87 \pm 8.25$ & $10.84 \pm 1.10$ & $7.15 \pm 0.83$ & n.d. & $40.53 \pm 4.54$ & $13.07 \pm 1.52$ & $0.73 \pm 0.13$ & & $0.33 \pm 0.02^{*}$ \\
\hline \multicolumn{10}{|l|}{ Hippocampus } \\
\hline WT & $74.10 \pm 8.20$ & $12.61 \pm 2.13$ & $4.64 \pm 0.83$ & n.d. & $32.83 \pm 4.61$ & $23.01 \pm 5.40$ & $0.44 \pm 0.08$ & & $0.71 \pm 0.11$ \\
\hline eNOS-/- & $89.82 \pm 7.69$ & $11.00 \pm 0.95$ & $5.65 \pm 0.29$ & n.d. & $35.48 \pm 2.64$ & $27.66 \pm 4.02$ & $0.53 \pm 0.03$ & & $0.76 \pm 0.06$ \\
\hline \multicolumn{10}{|c|}{ Ventral striatum } \\
\hline WT & $129.70 \pm 33.37$ & $764.51 \pm 68.02$ & $105.13 \pm 8.90$ & $80.18 \pm 8.35$ & $62.63 \pm 7.32$ & $13.34 \pm 2.40$ & $0.16 \pm 0.01$ & $0.10 \pm 0.01$ & $0.20 \pm 0.02$ \\
\hline eNOS-/- & $143.89 \pm 11.15$ & $677.66 \pm 34.97$ & $103.44 \pm 4.56$ & $89.13 \pm 6.90$ & $65.82 \pm 3.05$ & $17.20 \pm 1.79$ & $0.14 \pm 0.01$ & $0.13 \pm 0.01^{*}$ & $0.26 \pm 0.02^{*}$ \\
\hline \multicolumn{10}{|c|}{ Dorsal striatum } \\
\hline WT & $27.05 \pm 6.90$ & $1818.39 \pm 76.61$ & $198.44 \pm 11.77$ & $116.24 \pm 12.73$ & $37.27 \pm 4.15$ & $20.63 \pm 3.04$ & $0.11 \pm 0.01$ & $0.06 \pm 0.01$ & $0.53 \pm 0.03$ \\
\hline eNOS-l- & $38.81 \pm 14.52$ & $1948.69 \pm 71.37$ & $220.32 \pm 7.39$ & $111.50 \pm 18.50$ & $34.38 \pm 3.48$ & $21.87 \pm 3.07$ & $0.11 \pm 0.01$ & $0.06 \pm 0.01$ & $0.62 \pm 0.03$ \\
\hline
\end{tabular}

NA, Noradrenaline; n.d., not detectable. ${ }^{*} p<0.05 ;{ }^{+} p \leq 0.1$ versus WT controls.

training (trial blocks, $F_{(1,46)}=25.9, p<0.001$ ) without an interaction between genotype and time $(p>0.1)$. Again, no effect of genotype on the distance moved or on swimming speed was found (distance moved, $F_{(1,46)}=0.52, p=0.476$; Fig. $3 B$ ) (swimming speed, $F_{(1,46)}=0.56, p=0.458$; Fig. $3 C$ ). Cued learning performance (sum of latencies) on day 2 was better than on every other last day of the previous water maze testing stages ( $t$ test, all inner-group $p$ values $<0.05$ ).

\section{Brain monoamines}

In water maze-trained eNOS $-/-$ mice, 5-HIAA was increased in the cerebellum ( $t$ test, $p=0.020$ ) (Table 2). Cerebellar DA and dopamine turnover (HVA/DA) showed a tendency to decrease ( $p=0.097$ for both cases). Furthermore, turnover rates for serotonin (5HIAA/5-HT) were increased in the frontal cortex and ventral striatum of eNOS $-1-$ mice (frontal cortex, $p=0.010$; ventral striatum, $p=0.049$ ). Dopamine turnover increased in the ventral striatum of eNOS-deficient mice (DOPAC/DA, $p=0.023$ ).

\section{DISCUSSION}

In the present study, functional inactivation of the gene encoding endothelial NO synthase induced profound behavioral changes in the C57BL/6 mouse. In the open field, the eNOS-/- animals were less active but showed no signs of impaired habituation. In the elevated plus-maze, eNOS $-/-$ mice showed an increase in anxiety-related parameters. In the water maze, eNOS $-/-$ animals found the hidden platform sooner than WT controls under different experimental conditions, indicative of improved learning and memory capacities. Furthermore, several parameters of brain monoaminergic activity were affected in water maze-trained eNOS $-/-$ animals.

\section{Activity and emotionality}

During the first and third exposure to the open field, eNOS-/mice showed less locomotion and rearing behavior than controls. These changes are comparable with results obtained by pharmacological elimination of the whole NO system, because NOS inhibition was found to decrease spontaneous and pharmacologically induced activity (Ohno and Watanabe, 1995; Sandi et al., 1995). On the other hand, the decrease in general activity seen in the open field might be partly induced by an increase in the probability of anxiety-driven passive avoidance behavior, as indicated by an increase in time spent in the corners of the open field by $\mathrm{KO}$ mice. In the elevated plus-maze, an anxiogenic-like effect of the eNOS-/mice was also evident, because the animals spent less time on the open and more time on the walled arms of the apparatus. Furthermore, ethologically based analysis of behavior revealed changes in risk assessment, scanning, and end exploring, also indicative of a general increase in anxiety (Cruz et al., 1994). Thus, the results of the present study are in line with recent pharmacological studies in which inhibition of NO synthesis had anxiogenic effects in the elevated plus-maze (Lino de Oliveira et al., 1997) and diminished the anxiolytic effects of chlordiazepoxide (Quock and Nguyen, 1992) and nitrous oxide (Caton et al., 1994).

\section{Learning and memory}

The relative amounts of rearing and locomotion exhibited during the second exposure to the open field indicate that habituation learning of the eNOS $-/-$ mice was comparable with that of controls. During the third exposure, only the WT mice showed an increase in horizontal and vertical activity compared with the second trial. Besides the possibility that the non-reincrease in activity observed in eNOS $-/-$ mice is related to an anxiety component, as discussed above, this effect can be interpreted in terms of superior memory for the formerly unknown surrounding in the eNOS $-/-$ mice.

The eNOS $-/-$ mice also showed superior performance in the water maze, indicative of an improvement in learning and memory capacities. During initial acquisition training, the mean time to reach the hidden platform was lower for eNOS-/- animals; during the extinction trial or spatial probe trial, the groups were comparable in terms of time spent in the vicinity of the platform position and the total distance traveled. Five days later, however, during long-term retention testing, a striking difference in latency to reach the platform was observed. The eNOS $-/-$ animals showed a clear superiority in reaching the platform during the first retention trial. This difference disappeared over the course of 2 d retention training. During reversal learning, the eNOS $-/-$ mice were again superior in learning to find the platform at a new location. During cued learning with the platform made visible, no between-group differences were observable, indicating that the results of the former stages in water maze testing were not mainly attributable to unspecific sensorimotor or motivational effects of the gene disruption.

The results of the present study contrast with recent studies centering on the effects of systemic or local pharmacological inhibition of NO synthesis on spatial learning. In these studies, NO inhibition induced impairments (Yamada et al., 1995) or had no effect (Blokland et al., 1999). Here, besides factors such as species differences or different behavioral protocols, the biochemical origin of NO and its microanatomical location might be an important variable. It had been shown that the targeted gene inactivation of the neuronal NOS isoform had decreased NMDA-stimulated cor- 
tical glutamate release, whereas endothelial NOS inactivation reduced NMDA-stimulated GABA release in several brain regions, including the hippocampus (Kano et al., 1998). This selectivity might be related to a differential synaptic distribution of the NOS isoforms as it is discussed for the hippocampal pyramidal cells and interneurons in which eNOS seems to be localized to pyramidal neurons, whereas nNOS is localized to GABAergic interneurons (Dinerman et al., 1994; O‘Dell et al., 1994; Kano et al., 1998). Thus, via the loss of NO as a retrograde messenger at the inhibitory synapse between GABAergic interneurons and pyramidal cells, the eNOS gene disruption might disinhibit hippocampal consolidation processes by the selective attenuation of GABAergic inhibition of pyramidal cell activity.

The present results do not support the hypothesis that endothelial NO synthase-derived NO is necessary for the strengthening of memory traces, as has been inferred from the results of recent in vitro studies examining the influence of the eNOS gene inactivation on hippocampal and neocortical long-term potentiation. In these studies, it was found that the eNOS gene disruption led to deficits in the induction of neocortical and hippocampal LTP by weak tetanic stimulation (Haul et al., 1999; Wilson et al., 1999). During the last decade, a direct relation between LTP and memory was questioned on the basis of anatomical and functional data (for review, see Amaral and Witter, 1989; Moser et al., 1993; McEachern and Shaw, 1996). The fact that our eNOS null mice displayed superior water maze performance and exhibited LTP deficits challenges the widely held assumption that LTP represents a necessary neural or molecular foundation of spatial memory storage processes.

Besides synaptic plasticity, non-neuronal processes might be influenced by the eNOS inactivation. In recent studies, eNOS was found in the cerebral vasculature and astrocytes but not in neurons (Stanarius et al., 1997; Demas et al., 1999; Wiencken and Casagrande, 1999). Therefore, behavioral effects of eNOS inactivation might depend on changes in cerebral blood flow (Endres et al., 1998) or are related to peripheral processes, such as coronary blood flow (Gödecke et al., 1998), body temperature control (Steiner et al., 1998), or muscular oxygen supply (Shen et al., 1995).

\section{Brain monoamines}

Brain monoaminergic systems are known to play important roles in processes underlying learning and memory. Lesions of the noradrenergic system can improve rodent water maze performance (Sirvio et al., 1991), whereas lesions of the dopaminergic system or blockade of serotonergic transmission in combination with cholinergic blockade impaired such performance (Gasbarri et al., 1996; Harder et al., 1996). In our eNOS-/- mice, concentrations of the serotonin metabolite 5-HIAA were increased in the cerebellum. Serotonin turnover was increased in the frontal cortex of eNOS $-1-$ mice. Furthermore, in the cerebellum of eNOS-/animals, DA content and DA turnover (HVA/DA) showed an tendency to decrease, whereas dopamine turnover (DOPAC/DA) in the ventral striatum of eNOS $-/-$ animals was increased. It was found recently that deficits in spatial learning performance, induced by chronic pharmacological inhibition of NO synthesis, are paralleled by decreases in hippocampal 5-HIAA content and 5-HIAA/5-HT ratio, cortical 5-HIAA/5-HT ratios, and an increase in striatal DOPAC content (Yamada et al., 1995). Based on the results of only these few studies, detailed considerations regarding the relationship between $\mathrm{NO}$, monoamines, and spatial orientation would be premature. However, because eNOS gene disruption led to increases in monoamine turnover in the ventral striatum, one could speculate that the behavioral effects of the eNOS gene disruption might be partly related to changes in monoaminergic correlates of anxiety and reward. Dopamine in the nucleus accumbens is centrally involved in reward processes (Kiyatkin, 1995). Because it is known that spatial orientation performance depends on the reinforcing properties associated with a successful solving of the task (Buresova and Bures, 1981), it might be taken into consideration that deficits as well as potentiations in learning and memory tasks, as observed in the present study, are attributable to changes in reinforcement related processes rather than in encoding or retrieval systems per se (Huston and Oitzl, 1989).

Furthermore, it was shown recently that serotonin, an important endogenous modulator of anxiety-related behavior (Graeff et al., 1996), is decreased in the ventral striatum of anxious rats, as observed in the elevated plus-maze (Schwarting et al., 1998). Because basal anxiety levels may be related to water maze performance (Montkowski et al., 1997), it might be taken into account that the effects of NO on performance in tests for learning and memory are related to changes in transmitter systems, which have been implicated in emotional processes.

\section{Conclusions}

The results of the present study reveal that the functional inactivation of the endothelial NO synthase gene induces profound behavioral changes in the mouse. Most striking, besides an increase in anxiety-related behavior, a clear improvement in Morris water maze performance was observed, which was related to changes in brain monoaminergic systems. Furthermore, behavioral changes might be related to a decreased reactivity of GABAergic neurotransmission or to non-neuronal processes, such as vascular functions. It remains to be determined whether this improvement depends on increased spatial learning capacity per se or whether eNOS gene disruption-induced changes in brain processes related to anxiety or reward might play an important role. To clarify these issues, studies with a high spatial orientation component but different reward contingencies (radial maze and spatial recognition) and different levels of anxiety induction are under way.

\section{REFERENCES}

Amaral DG, Witter MP (1989) The three-dimensional organization of the hippocampal formation: a review of anatomical data. Neuroscience 31:571-591.

Bannerman DM, Chapman PF, Kelly PAT, Butcher SP, Morris RGM (1994) Inhibition of nitric oxide does not impair spatial learning. J Neurosci 14:7404-7414.

Barnes NM, Sharp T (1999) A review of central 5-HT receptors and their functions. Neuropharmacology 38:1083-1152.

Blokland A, De Vente J, Prickaerts J, Honig W, Markereink-van Ittersum M, Steinbusch H (1999) Local inhibition of hippocampal nitric oxide does not impair place learning in the Morris water escape task in rats. Eur J Neurosci 11:223-232.

Buresova O, Bures J (1981) Reward improves working memory of rats in the radial maze. Physiol Behav 27:211-215.

Caton PW, Tousman SA, Quock R (1994) Involvement of nitric oxide in nitrous oxide anxiolysis in the elevated plus-maze. Pharmacol Biochem Behav 48:689-692.

Chapman PF, Atkins CM, Allen MT, Haley JE, Steinmetz JE (1992) Inhibition of nitric oxide synthesis impairs two different forms of learning. NeuroReport 3:567-570.

Cruz APM, Frei F, Graeff FG (1994) Ethopharmacological analysis of rat behavior on the elevated plus-maze. Pharmacol Biochem Behav 49:171-176.

Demas GE, Kriegsfeld LJ, Blackshaw S, Huang P, Gammie SC, Nelson RJ, Snyder SH (1999) Elimination of aggressive behavior in male mice lacking endothelial nitric oxide synthase. J Neurosci 19:C1-C5.

De Souza Silva MA, Mattern C, Häcker R, Nogueira PJC, Huston JP Schwarting RKW (1997) Intranasal administration of the dopaminergic agonists L-dopa, amphetamine, and cocaine increases activity in the neostriatum: a microdialysis study in the rat. J Neurochem 68:233-239.

Dinerman JL, Dawson TM, Schell MJ, Snowman A, Synder SH (1994) Endothelial nitric oxide synthase localized to hippocampal pyramidal cells: implications for synaptic plasticity. Proc Natl Acad Sci USA 91:4214-4218.

Du W, Harvey JA (1996) The nitric oxide synthesis inhibitor L-NAME facilitates associative learning. Prog Neuropsychopharmacol Biol Psychiatry 20:1183-1195.

Dun NJ, Dun SL, Förstermann U (1994) Nitric oxide synthase immunoreactivity in rat pontine medullary neurons. Neuroscience 59:429-445.

Endres M, Laufs U, Huang Z, Nakamura T, Huang P, Moskowitz MA, Liao JK (1998) Stroke protection by 3-hydroxy-3-methylglutaryl (HMG)-CoA reductase inhibitors mediated by endothelial nitric oxide synthase. Proc Natl Acad Sci USA 95:8880-8885.

Estall LB, Grant SJ, Cicala GA (1993) Inhibition of nitric oxide (NO) production selectively impairs learning and memory in the rat. Pharmacol Biochem Behav 46:959-962.

Faraci FM, Brian JE (1994) Nitic oxide and the cerebral circulation. Stroke 25:692-703. 
Gasbarri A, Sulli A, Innocenzi R, Pacitti C, Brioni JD (1996) Spatial memory impairment induced by lesion of the mesohippocampal dopaminergic system in the rat. Neuroscience 74:1037-1044.

Gödecke A, Decking UKM, Ding Z, Hirchenhain J, Bidmon HJ, Gödecke S, Schrader J (1998) Coronary hemodynamics in endothelial NO synthase knockout mice. Circ Res 82:186-194.

Graeff FG, Giumaraes FS, De Andrade TGC, Deakin JFW (1996) Role of 5-HT in stress, anxiety, and depression. Pharmacol Biochem Behav 54:129-141.

Hara H, Waeber C, Huang PL, Fujii M, Fishman MC, Moskowitz MA (1996) Brain distribution of nitric oxide synthase in neuronal or endothelial nitric oxide synthase mutant mice using [3H]L-NG-nitro-arginine autoradiography. Neuroscience 75:881-890.

Harder JA, Kelly ME, Cheng CH, Costall B (1996) Combined pCPA and muscarinergic antagonist treatment produces a deficit in rat water maze acquisition. Pharmacol Biochem Behav 55:61-65.

Haul S, Gödecke A, Schrader J, Haas HL, Luhmann HJ (1999) Impairment of neocortical long-term potentiation in mice deficient of endothelial nitric oxide synthase. J Neurophysiol 81:494-497.

Huston JP, Oitzl MS (1989) The relationship between reinforcement and memory: parallels in the rewarding and mnemonic effects of the neuropeptide Substance P. Neurosci Biobehav Rev 13:171-180.

Huang AM, Lee EH (1995) Role of hippocampal nitric oxide in memory retention in rats. Pharmacol Biochem Behav 50:327-332.

Kano T, Shimuzu-Sasamata M, Huang PL, Moskowitz MA, Lo EH (1998) Effects of nitric oxide synthase gene knockout on neurotransmitter release in vivo. Neuroscience 86:695-699.

Kidd EJ, Michel AD, Humphrey PPA (1995) Autoradiographic distribution of $[3 \mathrm{H}]$ L-NG-nitro-arginine binding in rat brain. Neuropharmacology 34:63-73.

Kiyatkin EA (1995) Functional significance of mesolimbic dopamine. Neurosci Biobehav Rev 19:573-598.

Lino de Oliveira C, Del Bel EA, Guimaraes FS (1997) Effects of L-NOARG on plus-maze performance in rats. Pharmacol Biochem Behav 56:55-59.

Manzoni O, Prezeau L, Marin P, Deshager S, Bockaert J, Fagni L (1992) Nitric oxide-induced blockade of NMDA receptors. Neuron 8:653-662.

McEachern JC, Shaw CS (1996) An alternative to the LTP orthodoxy: a plasticity-pathology continuum model. Brain Res Rev 22:51-92.

Montague PR, Gancayco CD, Winn MJ, Marchase RB, Friedlander J (1994) Role of NO production in NMDA receptor-mediated neurotransmitter release in cerebral cortex. Science 263:973-977.

Montkowski A, Poettig M, Mederer A, Holsboer F (1997) Behavioural performance in three substrains of mouse strain 129. Brain Res 762:12-18.

Moser E, Mathiesen I, Andersen P (1993) Association between brain temperature and dentate field potentials in exploring and swimming rats. Science 259:1324-1326.

O`Dell T, Huang PL, Dawson TM, Dinerman JL, Synder SH, Kandel ER, Fishman MC (1994) Endothelial NOS and the blockade of LTP by NOS inhibitors in mice lacking neuronal NOS. Science 265:542-546.

Ohno M, Watanabe S (1995) Nitric oxide synthase inhibitors block behavioral sensitization to metamphetamine in mice. Eur J Pharmacol 275:39-44.

Quock RM, Nguyen E (1992) Possible involvement of nitric oxide in chlordiazepoxide-induced anxiolysis in rats. Life Sci 51:PL255-PL260.

Rolls ET (2000) Memory systems in the brain. Annu Rev Psychol 51:599-630.

Sandi C, Venero C, Guaza C (1995) Decreased spontaneous motor activity and startle response in nitric oxide synthase inhibitor-treated rats. Eur J Pharmacol 277:89-97.

Schwarting RK, Thiel CM, Müller CP, Huston JP (1998) Relationship between anxiety and serotonin in the ventral striatum. NeuroReport 9:1025-1029.

Shen W, Zhang X, Zhao G, Wolin MS, Sessa W, Hintze TH (1995) Nitric oxide production and NO synthase gene expression contribute to vascular regulation during exercise. Med Sci Sports Exerc 27:1125-1134.

Sirvio J, Riekkinen P, Valjakka A, Jolkkonen J, Riekkinen PJ (1991) The effects of noradrenergic neurotoxin, DSP-4, on the performance of young and aged rats in spatial navigation task. Brain Res 563:297-302.

Son H, Hawkins RD, Martin K, Kiebler M, Huang PL, Fishman MC, Kandel ER (1996) Long-term potentiation is reduced in mice that are doubly mutant in endothelial and neuronal nitric oxide synthase. Cell 87:1015-1023.

Stanarius A, Töpel I, Schulz S, Noack H, Wolf G (1997) Immunohistochemistry of endothelial nitric oxide synthase in the rat brain: a light and electron microscopical study using the tyramide signal amplification technique. Acta Histochem 99:411-429.

Steiner AA, Carnio EC, Antunes-Rodrigues J, Branco LG (1998) Role of nitric oxide in systemic vasopressin-induced hypothermia. Am J Physiol 275:R937-R941.

Wiencken AE, Casagrande VA (1999) Endothelial nitric oxide synthetase (eNOS) in astrocytes: another source of nitric oxide in neocortex. Glia 26:280-290.

Wilson RI, Yanovsky J, Gödecke A, Stevens DR, Schrader J, Haas HL (1997) Endothelial nitric oxide synthase and LTP. Nature 386:338.

Wilson RI, Gödecke A, Brown RE, Schrader J, Haas HL (1999) Mice deficient in endothelial nitric oxide synthase exhibit a selective deficit in hippocampal long-term potentiation. Neuroscience 90:1157-1165.

Yamada K, Noda Y, Nakayama S, Komori Y, Sugihara H, Hasegawa T, Nabeshima T (1995) Role of nitric oxide in learning and memory and in monoamine metabolism in the rat brain. Br J Pharmacol 115:852-858. 